\title{
Genomic and Transcriptomic Analyses of Bioluminescence Genes in the Enope Squid Watasenia scintillans
}

\author{
Masa-aki Yoshida ${ }^{1}$ (1) Junichi Imoto ${ }^{2,3} \cdot$ Yuri Kawai $^{4} \cdot$ Satomi Funahashi ${ }^{4} \cdot$ Ryuhei Minei $^{5} \cdot$ Yuki Akizuki $^{5}$. \\ Atsushi Ogura ${ }^{5} \cdot$ Kazuhiko Nakabayashi $^{6} \cdot \mathrm{Kei} \mathrm{Yura}^{4,7} \cdot{\mathrm{Kazuho} \mathrm{Ikeo}^{2}}^{2}$
}

Received: 8 April 2020 / Accepted: 28 September 2020 / Published online: 24 October 2020

(C) The Author(s) 2020

\begin{abstract}
Watasenia scintillans, a sparkling enope squid, has bioluminescence organs to illuminate its body with its own luciferase activity. To clarify the molecular mechanism underlying its scintillation, we analysed high-throughput sequencing data acquired previously and obtained draft genome sequences accomplished with comparative genomic data among the cephalopods. The genome mapped by transcriptome data showed that (1) RNA editing contributed to transcriptome variation of lineage specific genes, such as W. scintillans luciferase, and (2) two types of luciferase enzymes were characterized with reasonable 3D models docked to a luciferin molecule. We report two different types of luciferase in one organism and possibly related to variety of colour types in the W. scintillans fluorescent organs.
\end{abstract}

Keywords Firefly squid $\cdot$ Cephalopod $\cdot$ RNA editing $\cdot 3$ D modelling $\cdot$ Repetitive elements

\section{Introduction}

Around the Japanese sea, especially off Toyama, the appearance of shoals of the sparkling enope squid, Watasenia scintillans, heralds the beginning of spring. The W. scintillans is also known as the "firefly squid," which is important for both tourism and the fishing industry in Japan. W. scintillans emits light from three different types of photophores (Inamura et al. 1990). Five light organs are located around each eye, and a cluster of three light organs is located at the tip of the arms.

Electronic supplementary material The online version of this article (https://doi.org/10.1007/s10126-020-10001-8) contains supplementary material, which is available to authorized users.

Masa-aki Yoshida

mayoshida@life.shimane-u.ac.jp

Kei Yura

yura.kei@aoni.waseda.jp

1 Marine Biological Science Section, Education and Research Center for Biological Resources, Faculty of Life and Environmental Sciences, Shimane University, Oki, Japan

2 Department of Genomics and Evolutionary Biology, National Institute of Genetics, Mishima, Japan
The squid also has dermal light organs producing ambient, greenish light. The dermal light organs are utilized for counter-illumination camouflage to match the brightness making it difficult for predators below to detect the squid (Young and Roper 1977). Young et al. (1979) reported that Enoploteuthis, the closest relative of W. scintillans, has dermal light organs similar to the ones of $W$. scintillans. The members of the Enoploteuthidae seems to have a common mechanism of bioluminescence. Several researches in the past have established the molecular mechanism of illumination that 
coelenterazine disulfate is the luciferin substrate, and the reaction requires ATP, $\mathrm{Mg}^{2+}$ and molecular oxygen (Tsuji 2002; Tsuji 2005; Teranishi and Shimomura 2008; Goto et al. 1974; Inoue et al. 1975; Inoue et al. 1976).

In addition to the study of luminescence, cephalopod molluscs, especially squids, have fascinated many researchers studying not only in the field of fishery science but also in the field of molecular biology, especially neuroscience, visual physiology and biophysics (Schwiening 2012; Seidou et al. 1990; Hara and Hara 1980; Shichida and Matsuyama 2009; Murakami and Kouyama 2008). To fulfil the demand of the resources, the sparkling enope squid has played a decent role because collection of the squid is exceptionally stable compared with other deep-sea squids that are usually difficult to collect. However, the recent advance genome biology has not blessed this species.

Lack of genome information had prevented us from understanding the genetic basis of cephalopod biology. The report of octopus genome from Albertin et al. (2015) has finally advanced the understanding of Cephalopoda class. The genome analysis revealed three notable characteristics of the octopus genome: (1) highly rearranged genome with transposable element expansion, (2) lineage specific duplication of certain types of genes and (3) whole transcript-wide adenosine to inosine (A-toI) RNA editing (Alon et al. 2015; Liscovitch-Brauer et al. 2017). These characteristics featured the octopus genome as quite different from metazoan genomes. However, it is not clear whether these characteristics are specific to octopuses or to cephalopods including squids and cuttlefish. Squid and cuttlefish, different major lineage of the cephalopods, show similar body plans and morphology to the octopuses (Young 1971) but diversified over 270-284 million years ago (Mya) (Kröger et al. 2011; Vinther et al. 2012). The timescale is comparable with the time of the appearance of dinosaurs or mammals (324.7 Mya, dos Reis et al. 2015). Hence, comparative genomic studies among octopus, squid and cuttlefish may accentuate cephalopod commonalities with differences to other molluscs and spotlight the specific features among them.

The average genome size of the cephalopods known to date is slightly bigger than that of other molluscan species (Gregory 2019). We have estimated the genome size of the enope squid by comparing the mean copy number of mitochondria per cell and found that the haploid genome size was about 4.78 giga base pair (Gbp) (Hayashi et al. 2016). The size is bigger than the known genome sizes of squids (Gregory 2019). Recent genome analysis has revealed that the regions with three giga base in total in the octopus genome have been the result of enormous expansion of repetitive sequences, such as transposons, contributing nearly $45 \%$ of the genome (Albertin et al. 2015). Such repetitive elements (REs) often cause genomic rearrangement across the chromosomes
(Oliver and Greene 2009). For instance, the inserts of REs were estimated as a cause of disruption of macrosynteny of the genome and resulted in the loss of the HOX gene cluster in octopus (Albertin et al. 2015). The question to be addressed is how REs arose and expanded across the cephalopod genomes during the evolution. Typically, types of causal transposable elements (TEs) show the history that the species has followed and are related to the characteristics of the organism. For example, Alu-type short interspersed nuclear element (SINE), which is the most abundant repeat sequence found in human genome, occupies more than $10 \%$ of the genome sequence (Lander et al. 2001). Despite the abundance, a major burst of the Alu amplification was estimated to have happened 25 50 Mya based on the human Alu subfamily sequence diversity (Shen et al. 1991). TEs are the major part of REs in the cephalopod genomes (da Fonseca et al. 2020) and undergo a rapid increase in copy number in some animal clades, resulting in the increase of the genome size through TE amplification and, in some cases, in causing loci rearrangements through TE insertions. The former was already observed in squid and in octopus (Yoshida et al. 2011; Albertin et al. 2015), and the latter was suggested to have happened as large-scale genomic rearrangements in the genome of California two-spot octopus Octopus bimaculoides (Albertin et al. 2015). It is considered that the timing of its appearance is greatly related to the reorganization of the genome; however, burst timing of REs are uncertain in the cephalopods even before/after squid-octopod divergence.

Another characteristic of cephalopod genetic system is the existence of extensive RNA editing (Liscovitch-Brauer et al. 2017). The events of RNA editing itself have been discovered in wide range of organisms including humans, Drosophila and so forth. However, numerous RNA-editing sites were conserved between squids and octopuses, especially in transcripts of nervous system, which is quite contrary to the situation in mammals and Drosophila. In humans, for instance, the locations of RNA editing in only 25 genes are conserved across mammals (Pinto et al. 2014). In Drosophila, only about 65 editing sites are conserved across the Drosophila lineage (Yu et al. 2016). RNA editing is expected to be utilized for enhancing transcriptome variation (Liscovitch-Brauer et al. 2017).

Recently, the genome of Hawaiian bobtail squid Euprymna scolopes, a model cephalopod with symbiotic luminescence, has been sequenced, and the highly repetitive nature of the genome was uncovered (Belcaid et al. 2019). To solidify the characteristics of cephalopod genomes and to uncover the commonality of RNA editing in this group of organisms, we obtained the draft genome sequences of the Japanese enope squid, W. scintillans, which has several light organs different from the ones of E. scolopes. We further used a genomic approach in combination with transcriptomic approach, namely assembly of both DNA and RNA sequences, and performed 
comparative genomics among the cephalopods. Based on these comparative genome analyses, two characteristics of cephalopod genomes have been confirmed: (1) RE variation in W. scintillans is different from that of octopus, and (2) RNA editing was contributed to transcriptome variation of lineage specific genes, such as $W$. scintillans luciferase. Subsequent detailed analysis of bioluminescence showed that (3) there are at least two types of luciferases in the genome of $W$. scintillans.

\section{Materials and Methods}

\section{Sample Collection and Isolation of Genomic DNA and Total RNA}

W. scintillans caught in Toyama Bay were obtained from Hotaruika Aquarium (Namerikawa, Toyama, Japan). Genomic DNA was extracted from the brain of a single female individual (L3) by a conventional phenol/chloroform extraction protocol. Briefly, the brain tissue was lysed in $600 \mu \mathrm{l}$ of the lysis buffer $(0.1 \mathrm{M}$ Tris- $\mathrm{HCl} \mathrm{pH} 8.0,0.2 \mathrm{M} \mathrm{NaCl}, 5 \mathrm{mM}$ EDTA, $0.2 \%$ SDS, $0.2 \mathrm{mg} / \mathrm{ml}$ Proteinase $\mathrm{K}$ ) at $55^{\circ} \mathrm{C}$ overnight. The lysate was extracted with an equal volume of phenol/chloroform/isoamyl alcohol $(25: 24: 1)$ once, and with an equal volume of chloroform once. The genome DNA was precipitated by adding an equal amount of isopropanol to the lysate, washed with $70 \%$ ethanol, dried briefly and dissolved in $1 \times$ TE. Total RNA samples were also extracted from the brain and the arm tips of the same individual using the Mixer Mill MM301 (Retsch, Düsseldorf, German) for homogenization and the E.Z.N.A. Mollusc RNA Kit (Omega Bio-tek, Georgia, United states).

\section{Library Preparation and Sequencing}

A short-insert and two mate-pair libraries were prepared from the brain genomic DNA of an individual using TruSeq DNA sample preparation kit v2 (Illumina, San Diego, USA) and Nextera mate-pair library preparation kit (Illumina), respectively. Mate-pair libraries were prepared from two size ranges of tagmented DNA fragments, $4 \mathrm{~kb}$ and $8 \mathrm{~kb}$ in average. RNA-seq libraries were prepared from the total RNA samples of the same individual (brain and arm tips) using TotalScript RNA-Seq Kit (Epicentre, Wisconsin, USA). The DNA- and RNA-seq libraries were sequenced on HiSeq1500/2500 systems (Illumina). Paired-end read $(150 \mathrm{bp} \times 2)$ sequencing was performed using the HiSeq Rapid SBS Kit HS. The conversion of bcl files to fastq files was performed using the CASAVA v1.8.2 (configureBclToFastq.pl) software (Illumina).

\section{Genome and Transcriptome Assembly}

After multistage base trimming, 159.3 million read pairs from the short-insert ( $350 \mathrm{bp}$ ) library and 22.7 million and 23.4 million read pairs from two mate-pair libraries $(4 \mathrm{~kb}$ and $8 \mathrm{~kb})$ were subjected to 17-mer counting of the Illumina reads using Jellyfish v.2.2.10 (Marçais and Kingsford 2011). The k-mer histogram was processed using GenomeScope v1.0 (Vurture et al. 2017) to estimate genome size, heterozygosity and repeat content. All the same reads were also applied to de novo genome assembly using ALLPATHS-LG v.44837 (Gnerre et al. 2011) to generate genome contigs. Transcriptome assembly was performed by the CLC Assembly Cell (QIAGEN Bioinformatics, Hilden, Germany) independently to the draft genome. The single copy genes were assessed by BUSCO v1.1b1 with lineage option, metazoa (Simão et al. 2015). The assembled partial genome sequences were deposited in the DNA DataBank of Japan (DDBJ) under the accession numbers BLWP01000001-BLWP01491107. Raw Illumina reads for genome and transcriptome are available in the DDBJ Sequence Read Archive (DRA) under BioProject accession number PRJDB8630 and DRA accession number DRA009937, respectively. Augustus v3.2 was employed to generate an ab initio gene models (16,509 genes) with species option, human (Stanke et al. 2008). The protein gene model file is available as a supplementary file.

\section{Repeat Estimation}

To detect RE sequences from the molluscan whole genome shotgun sequences, we utilized the RepeatMasker programme (http://www.repeatmasker.org, RepBase Update 13.04; Kohany et al. 2006). To identify de novo REs from the molluscan genomes, the quality-controlled sequences were repeat-masked using the RepeatMasker with species-option set as "eukaryotes" due to the absence of a molluscan repeat database. For the RE database, we utilized owl limpet Lottia gigantea (Simakov et al. 2013), O. bimaculoides (Albertin et al. 2015) and Japanese pygmy squid Idiosepius paradoxus (Yoshida et al. 2011), respectively. As a comparison, the proportions of the three repeat sequences of three cephalopod genomes, $O$. bimaculoides, East Asian common octopus $O$. sinensis (https://www.ncbi. nlm.nih.gov/genome/84214?genome_assembly_id=678426) and giant squid Architeuthis dux (da Fonseca et al. 2020), were analysed using the same procedures.

\section{RNA Editing Analysis}

We mapped RNA-seq reads to the $W$. scintillans genome by TopHat (v2.1.0, Trapnell et al. 2009), and identified SNPs by SAMtools (v0.1.19, Li et al. 2009). We also identified polymorphic positions from the SNPs predicted above by GATK Haplotype Caller (v3.5, DePristo et al. 2011) with the default 
setting of the discovery mode. We implemented kallisto to estimate expression levels of RNA editing enzymes ADAR genes of each sample (Bray et al. 2016). We then extracted RNA editing site candidates where the sites show polymorphic but not SNPs by BEDTools (v.2.17.0, Quinlan and Hall 2010). We finally predicted RNA editing sites by REDItools (v1.0.4, Picardi and Pesole 2013), the curation of RNA editing sites investigation software. Numbers of RNA editing sites shared between brain and arm samples are estimated with a custom script and shown in a Venn diagram (Figure S10).

\section{Orthologous Analysis}

We utilized W. scintillans gene models estimated above for the orthologous relationship analysis. We also obtained gene models of $O$. bimaculoides and L. gigantea from the public databases. To estimate orthologous groups, we utilized OrthoFinder (v1.0.6, Emms and Kelly 2015) that can produce orthologous group of genes in which lineage specific duplicated genes would be included in the group.

\section{Search for Bioluminescent Proteins}

We utilized homology search for finding bioluminescent genes. We first conducted extensive search of scientific literature, internet description and databases for known bioluminescent proteins and ligands. The following are the database accession numbers either from UniProt or GenBank of the ten non-homologous representatives from bioluminescent protein groups with their origin: hetero dimer of P23146 and P19840 from a gammaproteobacterium Photorhabdus luminescens, P08659 from common eastern firefly Photinus pyralis, C6KYS2 from purpleback flying squid Sthenoteuthis oualaniensis, AAA29804 from sea pansy Renilla reniformis, CAA49754 from gregarious jellyfish Clytia gregaria, BAG48250 from a copepod Metridia pacifica, O77206 from a photosynthetic dinoflagellate Lingulodinium polyedrum, P17554 from sea-firefly Vargula hilgendorfii, Q9GV45 from a luminous shrimp Oplophorus gracilirostris and CAA10293 from common piddock Pholas dactylus. We performed a homology search of the whole amino acid sequences derived from the genome and transcriptome data of $W$. scintillans for these eleven amino acid sequences using BLAST with a cutoff of E-value $<10^{-5}$ and the score $>90$.

\section{Phylogenetic Analysis of Luciferases}

To figure out $W$. scintillans luciferases within the animal luciferases, wsluc1-4 and symplectin-like gene from Watasenia, as well as adding all sequences from one of the fireflies (firefly beetles (taxid:7049)), all drosophila sequences, all human sequences, all octopus sequences (Octopodiformes (taxid:215451)), all squid sequences (Decapodiformes (taxid:215450)), all Lottia sequences and transcripts from Branchiostoma, Crassostrea, Mytilus and Nematostella invertebrate adenylating enzymes were found by the NCBI web BLASTP search (as of 28 August 2020) using the $W$. scintillans luciferases as a query (e-value $<1.0 \times 10^{-30}$ ). To perform multiple alignment of protein sequences, we utilized MUSCLE v3.8.1551 (Edger 2004) followed by removing suspicious residues using trimAl_v1.4beta (automated1 option; Capella-Gutiérrez et al. 2009). RaxML-NG-mpi (RAxML-NG v. 0.9.0 released on 20.05.2019, Kozlov et al. 2019) was implemented to estimate the maximum likelihood tree with all option and the best fit model tested with modeltest-ng v. 0.1.3 (WAG+G4, Darriba et al. 2020). The tree was visualized with FigTree v1.4.2 (Rambaut 2009).

\section{Protein Three-Dimensional Structure Modelling}

A set of candidate proteins for bioluminescence identified by homology search went through homology modelling to test the structural compatibility for the luminescence function. The template protein structure of each group for the homology modelling was selected from Protein Databank (PDB) (Kinjo et al. 2018) by searching the homologue of the representative protein. Then each candidate protein sequence was aligned with the amino acid sequence of template protein using ALAdeGAP (Hijikata et al. 2011), the alignment tool specifically developed for homology modelling. Based on the alignment and the three-dimensional (3D) structure of the template protein, ten 3D structures of the candidate protein were build using MODELLER (Šali and Blundell 1993). Based on DOPE energy in MODELLER, the best structure out of the ten was selected and the reasonability of the selected 3D structure was tested on ProSA-web (Wiederstein and Sippl 2007), a tool to check the compatibility of the structure against the whole $3 \mathrm{D}$ structures in PDB. When the template 3D structure had luciferin or its analogue, then the molecule was docked to the modelled structure by superposing the template and target structures and was transferred the coordinates of the molecule from the template to the target structures. The reasonability of the location of the luciferin was examined by the existence of atomic clash between the protein and luciferin. When only few clashes exist between the protein and luciferin, then the structure was interpreted as reasonable.

\section{Results}

\section{Genome Assembly Statistics}

Total read obtained in this study ( $117 \mathrm{G}$ base pairs) gives more than $24 \times$ coverages compared with the estimated haploid genome size, $4.78 \mathrm{Gbp}$ (Table S1-S2, Hayashi et al. 2016). However, de novo genome assembly unexpectedly resulted 
in partial genome sequences with only $649 \mathrm{Mbp}$. But we conjectured that the data is still valuable for performing a large-scale comparison of genome components among molluscs (Table S3). To confirm the genome size estimation, we performed k-mer index analysis with Jellyfish and GenomeScope. Genome unique length size estimates from the GenomeScope were 666 to $672 \mathrm{Mbp}$, in reasonable agreement with those obtained by Allpaths-LG assembly (Figure S1). The analysis using GenomeScope also indicated high heterozygosity (4.9-5.9\%; Figure S1), which is consistent with the difficulty of assembly by short read. Genome haploid length estimates were 2.32 to $2.34 \mathrm{Gbp}$. This is equivalent to 18.7 in terms of k-mer coverage, which contradicts the earlier coverage estimate. We further searched for the signatures of singleton genes, which should exist in the sequence and found HOX coding sequences in our data. Four scaffolds showed significant similarity to amino acid sequences of known HOX gene cluster members (Antenapedia on scaffold_238533, Hox3 on scaffold_2002, Hox5/Sex comb reduced on scaffold_88306 and Posterior2 on scaffold_309777). On each scaffold, 11, 12, 22 and 11 raw genome reads were mapped, respectively (Table S4; Figure S2). On average, the genome data showed 11.5 times coverage. This value was also supported by the distributions of 576 singleton genes based on the BUSCO metazoa, which has a peak around 12-14 times coverage (Figure S3). Since this value is almost one and a half times by the estimates of the $\mathrm{k}$-mer index, the k-mer estimates are inferred to include both heterozygous and homozygous regions. The discrepancy in the number of coverage (either 24 or 11.5) likely derived from high heterogeneity among chromosomes and among the noncoding repetitive regions, which is shown below. High heterogeneity is also expected from fewer N50 (Table S3). The assembly is still highly fragmented, but the coding regions was apparently well covered and hence can be used for protein-coding gene analyses including estimation of the amount of RNA editing sites.

\section{Repeat Variations}

To detect RE sequences and their frequency in the genome, we applied RepeatMasker, a homology-based method, on the assembly. Repeat detections were performed in the following three steps. (1) We detected simple repeats and repeat common to metazoan in the $W$. scintillans assembly by default setting (for human) and masked. Then, (2) the repeat libraries of octopus at OIST Octopus Genome site (https://groups.oist. $\mathrm{jp} / \mathrm{ja} /$ molgenu/octopus-genome) were used as templates, and (3) the repeat libraries of I. paradoxus repeat database which we published previously (Yoshida et al. 2011) were used. By applying method 1 , we estimated that the total repeat content accounted for $16.69 \%$ (10.8 Mb) of the assembly (Table S5), dominated by simple repeats. By further applying method 2,
$5,149,360$ bp in $W$. scintillans genome (approximately $0.79 \%$ ) were estimated to comprise the repetitive sequence common to Coleoidea. By finally applying method 3, 12,458,350 bp sequences $(19.2 \%)$ on $W$. scintillans scaffolds were still found to have high similarity to the I. paradoxus REs. The abundant number of REs after the third screening step indicates that the relative volume of REs common to squids outnumbered REs in octopus (Fig. 1; Table S5). On the other hand, the REs found in the genomes of the two octopus species are heavily biased towards the octopus common repeat library and have small contribution of Idiosepius library. Although Architeuthis is a member of oegopsid squids and the most closely related group to $W$. scintillans among the animals whose genomes are now available, the genome has a distinct pattern of REs, possibly reflecting different RE distribution or maybe the assembly procedure (Fig. 1). The amount of repeat contents given here are likely underestimated due to the incompleteness of both the assembly of the genome sequence and the contents of I. paradoxus repeat database.

\section{Orthologous Gene Analysis}

To illuminate orthologous gene distribution among cephalopods, we first estimated orthologous groups of genes using W. scintillans, O. bimaculoides and L. gigantea as an outgroup species. As a result, we found 9535, 11,300 and 9817 orthologous gene groups in W. scintillans, $O$. bimaculoides and L. gigantea, respectively (Figure S4). Singlet genes that were found only in a specific species were not used in the following analyses. In the investigation of orthologous gene groups among the three species, we found that about $70 \%$ of the genes are conserved in molluscs. There are several percent of genes shared between $O$. bimaculoides and L. gigantea, but not in W. scintillans (2284 common genes between $O$. bimaculoides and L. gigantea compared with 493 ones between W. scintillans and L. gigantea; Figure S4). We rather speculated that this was caused by the incompleteness in the $W$. scintillans. Therefore, we continue the following analyses from the standpoint of $W$. scintillans assembly and will not touch upon the issue of missing genes in $W$. scintillans genome. From this viewpoint, we can conclude that more than $90 \%$ of the gene groups (7003) are shared in cephalopods. Of these gene groups, we examined the sequence diversity of 1:1:1 core orthologous genes from the divergence point of $W$. scintillans and $O$. bimaculoides by setting L. gigantea as an ancestor. The distribution of sequence diversity deduced from the length of branches on each phylogenetic tree seems similar, but sequence diversity of the $O$. bimaculoides genes is slightly wider than those of the W. scintillans (Figure S5). We also conducted functional enrichment analysis of squid-specific duplicated genes in the 7003 common orthologous groups for molluscs and found that signalling pathway genes including $\mathrm{G}$ protein coupled 
Fig. 1 RE frequency comparison of three cephalopod genomes

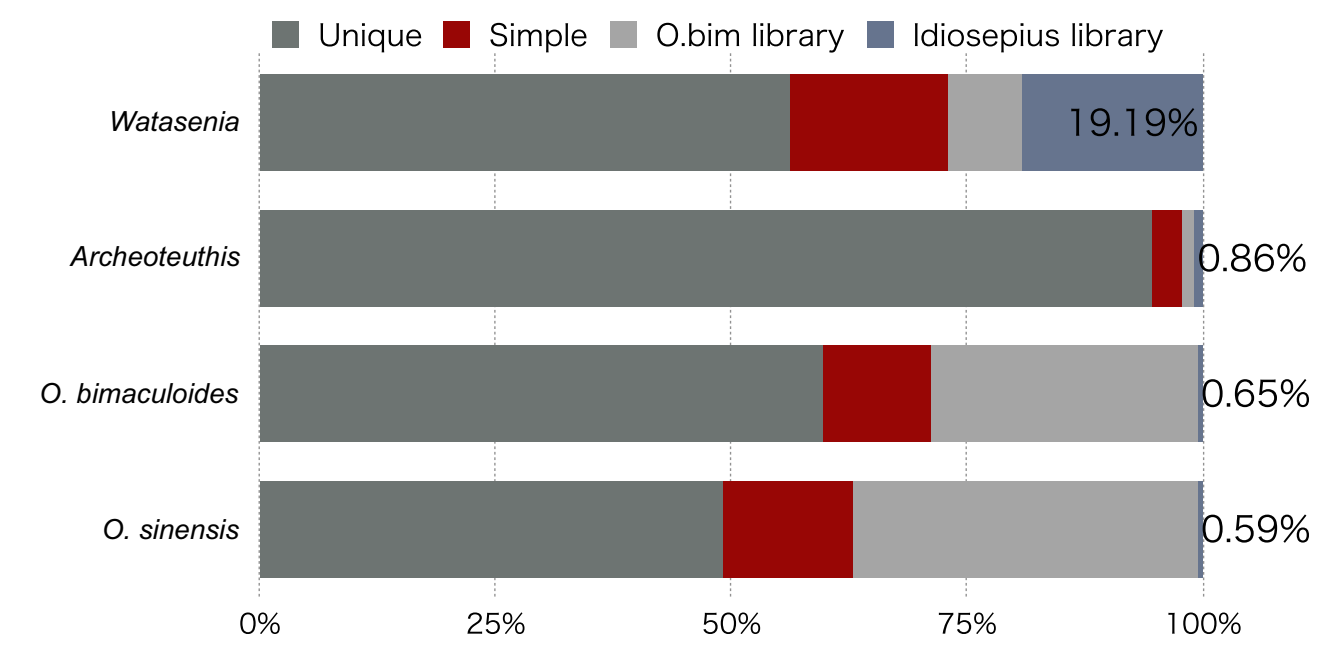

receptors have been enriched in enope squid, probably reflecting their characteristics.

\section{Bioluminescence Genes}

We searched for luciferase candidates from the W. scintillans genome assembly, brain RNA transcriptome and arm tip RNA transcriptome. We obtained two candidate partial sequences from brain transcriptome and four candidate partial sequences from the assembly and arm tip transcriptome. The former two sequences were very similar to the proteins reported as W. scintillans luciferase by Gimenez et al. (2016). Gimenez et al. reported four sequences (wsluc 1, 2, 3, 4) as candidates of $W$. scintillans luciferase or enope squid luciferase derived from the RNA transcriptome of light organ in the arm tip. One of the sequences has already been registered in GenBank (Accession number: LC177398). The sequence was similar to Photinus pyralis (firefly) luciferase. The two sequences we found in the brain RNA transcriptome were also similar to $P$. pyralis luciferase, and hence our result strengthened the possibility that the protein is indeed the luciferase of W. scintillans (Figures S6, S7). To figure out W. scintillans luciferases within the animal luciferases, we perform phylogenetic analysis with insect luciferase genes obtained from Fallon et al. (2018) (Figure S8). The W. scintillans luciferases made a clade sister to firefly luciferases characterized by Fallon et al. (2018).

The latter four candidates derived from genome assembly and arm tip RNA transcriptome had very similar sequences to S. oualaniensis symplectin. S. oualaniensis has been known to have another type of squid autofluorescence, which gives off shiny blue light (Tsuji and Leisman 1981; Chou et al. 2014). Fujii et al. (2002) singled out the agent of S. oualaniensis light and found symplectin as a new luciferase. Symplectin is similar to mammalian enzymes named biotinidase that hydrolyses biocytin to biotin and lysine (Cole et al. 1994). The sequence alignment between symplectin and the four candidates is

shown in Fig. 2a. The enzymatic detail of symplectin is still unknown, but its human homologue vanin-1 is well studied. The 3D structure of vanin- 1 has been determined with pantetheine analogue inhibitor, RR6 (Boersma et al. 2014). The putative catalytic sites of the enzyme reside on the amino acid residues pointed by red triangle in Fig. 2a. These residues correspond to Glu60, Lys163 and Cys196 in symplectin. In addition to these three residues, Cys390 is specifically important for symplectin where coelenterazine forms covalent bond (Isobe et al. 2008). Four sequences we found in this study were all partial sequences compared with the full-length symplectin, and none of the four sequences had the Cterminal domain of symplectin. Two of the four sequences lack the first catalytic residue Glu60. We speculate that these four sequences are partial sequences identified either in the incomplete genome assembly or RNA transcriptome because the identified sequences were highly similar to the N-terminal domain of symplectin and the model 3D structures of the proteins formed reasonable structure $(Z=-5.99$ in ProSA) with RR6, which is expected to be coelenterazine on

Fig. 2 Partial sequences of symplectin-like proteins in Watasenia scintillans assembly (contig\# s249267, s334436, s464828) and in arm tip transcriptome (c144950). a Amino acid sequence alignment among Sthenoteuthis oualaniensis symplectin (PDB ID; C6KYS2), W. scintillans sequences and human vanin-1 (Uniprot Accession; $4 \mathrm{CYG})$. Red triangle is the location of a catalytic site on vanin-1. The number at the last of the alignment is the length of the sequence. $\mathbf{b}$ Predicted three-dimensional structure of s249267 based on the structure of human vanin-1. The sequence runs from blue to red. Three catalytic residues are shown in stick model. The structure of human vanin-1 was determined with RR6 inhibitor, which is shown in purple stick model. The catalytic residues are well aligned to RR6, which is expected to be replaced by coelenterazine in symplectin-like protein. s249267 does not have about 50 residues at the $\mathrm{N}$-terminal side and 240 residues at the $\mathrm{C}$ terminal side compared with human vanin-1. The $\mathrm{C}$-terminal side can be located in the yellow background region, and the residue corresponding to Cys 390 of $S$. oualaniensis symplectin can be located at the far side of the putative coelenterazine-binding site 
a

SYmplectin (C6KYS2) 1 YVRPPSSWKVAVFEHQVIPPKTD--METREEAL்DAKLNSDVŸHEAVLESRS s249267 s3344361 s464828 1 c144950 1 Vanin-1 (4CYG) 1

DVICEYYDNIQNVDCRSSNCG - VVIFQFLNVHFLKCSFFIVPFSSHILFFL KQDTFIAAVYEHAAILPNATLTPVSREEALALMNRNLDILEGAITSAAD -

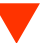

C6KYS2 s249267 s334436 s464828 c144950 4 CYG $\frac{1}{1}$ 1 51

50

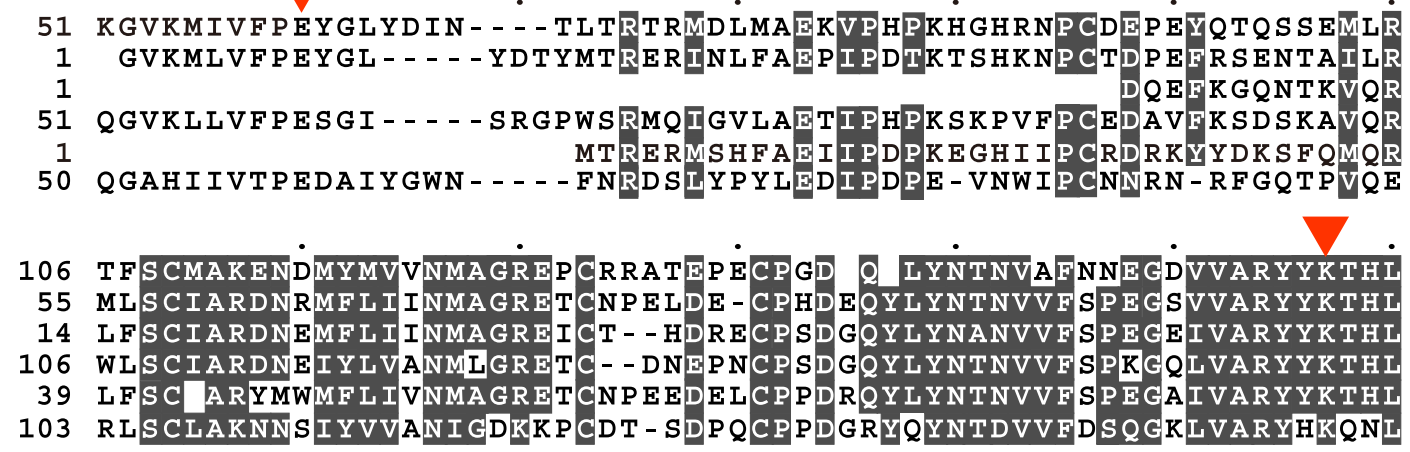

106 TFSCMAKENDMYMVVNMAGREPCRRATEPECPGD Q LYNTNVAFNNEGDVVARYYKTHL 55 MLSCIARDNRMFLIINMAGRETCNPELDE - C P HDEQYLYNTNVVFSPEGSVVARYYKTHI 14 LF S I ARDNEMFL I INMAGR ICT - - HDRECP S DGQYLYNANVVF S P EEIVARYYKTHL 106 WLSCIARDNEIYLVANMI GRETC - - DNEPNCP SDGQYLYNTNVV F S R GQLVARYYKTHI 39 LFSC ARYMWMFLIVNMA GRETCNPEEDELCP PDRQYLYNTNVVFSPEGAIVARYYKTHI 103 RLS CLAKNNS I YVVAN I GDKK PCDT - SD P QC P P D G RYQYNT DVVFDSQ GKLVAR YHKQNL

C6KYS2 s249267 s334436 s464828 C144950 4 CYG

C6KYS2 s249267 s334436 s464828 c144950 4 CYG

C6KYS2 s249267 s334436 s464828 c144950 4 CYG

\section{.}

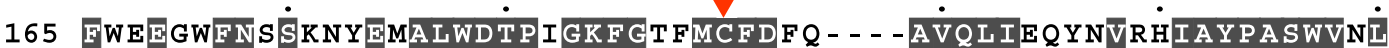
114 F-EFDWFNSAK SVEMA IFDTP F GKF GTFICFDIMFKEPTVELIKQHKIENIAYS SAWVEH 72 - GENWFNPAKEAEAAIFDTPFGKFATIICFDIMFENPIIELIEKYKIFNIAFPTAWVKL 164 F- GED SLD - PAEPEAAIFDTPFGKFATFICFDIVFKKPTIDLIEKYNIRNIAYPTAWVKI 99 S - K ENWFDAAKAS IAAIFDTPFGKLFVLMCFDVLFEKPAMELIQKHNIRNIAY P TASVDI 162 FMGENQFNVPKEPEIVTFNTTFG FGIFTCFDILFHDPAVTLVKDFHVDTIVFPTAWMNV

b

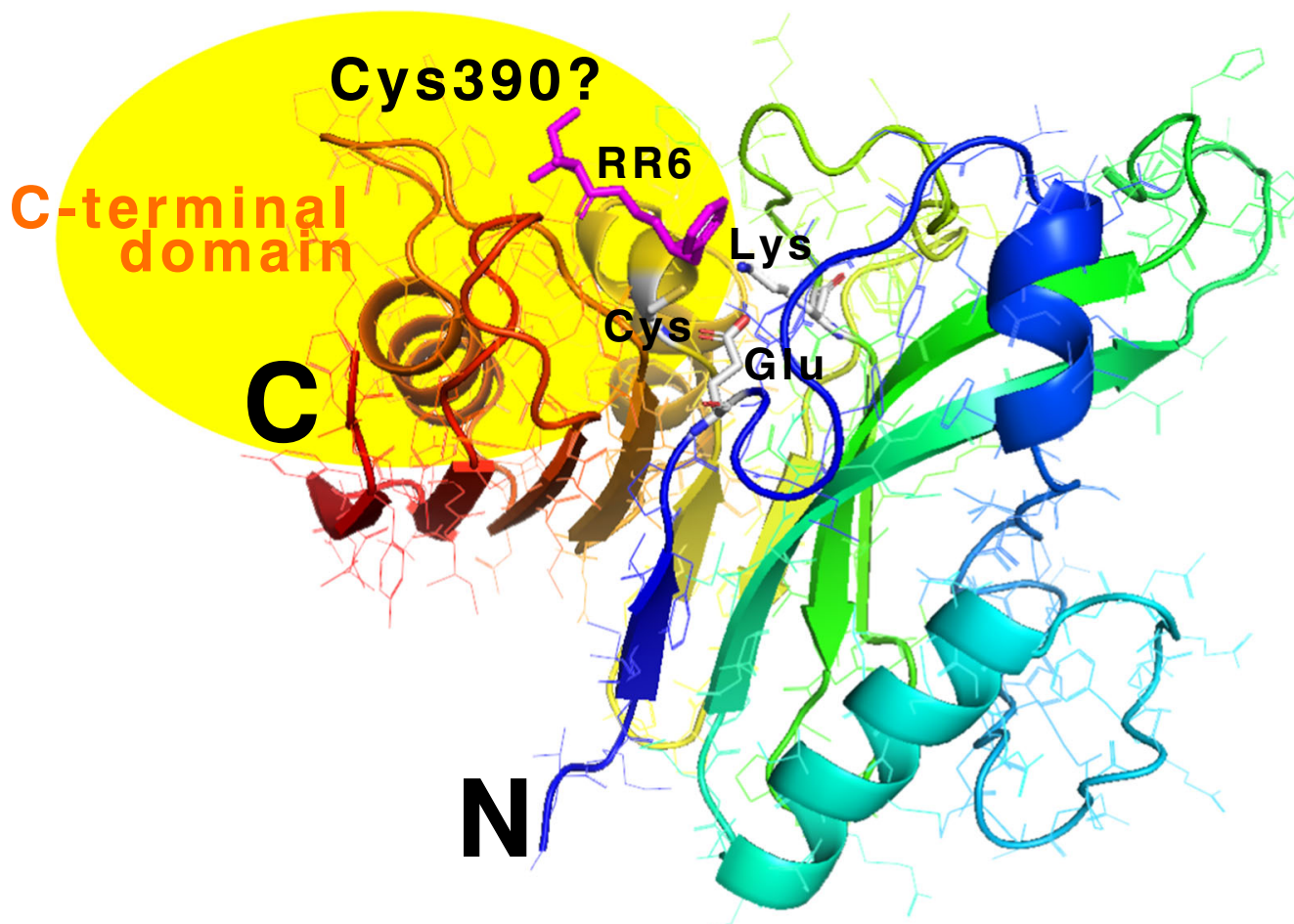


symplectin. Figure $2 \mathrm{~b}$ shows the model of a $W$. scintillans contig (\#s249267). The C-terminal domain with Cys390 should exist at the region depicted by yellow oval background in Fig. 2b, where part of the ligand protruded. Francis et al. (2017) has also found a single sequence similar to symplectin in the transcriptome data of W. scintillans obtained by Gimenez et al. (2016) and built a 3D structure. As in the first case in this study, the four sequences we found were also similar to $S$. oualaniensis symplectin, and hence our result strengthened the possibility of the existence of symplectinlike protein in $W$. scintillans.

\section{Extensive RNA Editing Events in the Luciferase}

RNA editing is a post-transcriptional mechanism that involves a substitution of a specific nucleotide at the RNA level, and substitution of A-to-I occurs preferably during the posttranscriptional stage (Bass and Weintraub 1988). The combination of genome sequencing and RNA-sequencing utilizing next generation sequencing technologies made us possible to investigate RNA editing sites with ease. In cephalopods, RNA editing is known to contribute to the temperature-sensitive responding mechanism in octopus (Garrett and Rosenthal 2012). Adenosine deaminases, ADAR1 and ADAR2, are known to proceed RNA editing and are conserved in varieties of bilateral animals. Therefore, we first checked gene expression activity of ADAR1 and ADAR2 in our transcriptome data of the brain and arm and found extensive expressions of those genes in the brain rather than arm, suggesting that RNA editing events do occur in $W$. scintillans and that the events are likely more active in the brain (Table 1). In the prediction of RNA editing sites, we mapped RNA-seq reads to the genome and found that more than ten thousand of sites experienced RNA editing. As we sequenced RNA with non-stranded library construction, T-to-C substitution as well as A-to-G substitution is dominant in the RNA editing site candidates (Figure S9). To investigate the tissue specificity of the RNA editing sites, we classified A-to-G RNA editing sites by Venn diagram and found that there is little coverage of RNA editing sites and the number of sites is greater in the brain (Figure S10).

The analyses showed that RNA editing was taken place extensively in $W$. scintillans. The finding of extensive RNA editing, especially on a novel species-specific genes of $W$. scintillans, here makes a new question arise, namely the extent of the rapidity in RNA editing evolution. To address this question, we scrutinized RNA editing in W. scintillans specific genes, the luciferases, characterized in this study. The luciferases are apparently forming a family with O. bimaculoides acyl CoA synthase. In the arm RNA transcriptome, 197 reads, giving approximately $21 \times$ coverage per site, were mapped onto the wsluc1 fragment on the W. scintillans genome (BLASTN, $>95 \%$ identity). We found 13 reproducible mismatches between genome and RNA from the same individual. Out of 13 , three were A-to-G mismatches and 10 were cytosine to thymine mismatches. However, some RNA reads still supported A as in the DNA in those thirteen mismatch sites; therefore, those mismatches were likely the outcome of RNA editing but not complete edit sites. Those sites were close to each other and located within $30 \mathrm{bp}$. We found that two out of the three caused synonymous substitutions. An intriguing note should be taken here that wsluc1 was also expressed in the brain, but no RNA editing was found on the brain-expressed wsluc1 RNA molecule.

\section{Discussion}

\section{Amplification of the Repeat Element in Cephalopod Genomes}

In general, genome rearrangement across TEs was suggested to be taken place only after TE expansion. For example, analysis of primate synteny breakpoint highlighted the role of TE in partial duplication and ultimately resulted in chromosome rearrangement (Capozzi et al. 2012). In case of octopus, Albertin et al. (2015) reported that genes that are linked in other bilaterians but not in octopus are enriched in

Table 1 Expression intensities of RNA editing enzymes (ADARs)

\begin{tabular}{|c|c|c|c|c|c|c|c|}
\hline \multirow[t]{2}{*}{ Brain } & \multirow[t]{2}{*}{ tblastn } & \multirow[b]{2}{*}{ evalue } & \multirow[b]{2}{*}{ bitscore } & \multicolumn{3}{|l|}{ kallisto } & \multirow[b]{2}{*}{ TPM } \\
\hline & & & & length & eff_length & est_counts & \\
\hline ADAR1 & TRINITY_DN1369507_c1_g9_i1 & $8 \mathrm{E}-105$ & 350 & 2344 & 2135.93 & 2137.23 & 6.32754 \\
\hline ADAR2 & TRINITY_DN1372101_c0_g5_i3 & $2 \mathrm{E}-173$ & 513 & 1486 & 1277.93 & 211.139 & 1.0448 \\
\hline \multirow[t]{2}{*}{ Arm } & tblastn & & & kallisto & & & \\
\hline & & evalue & bitscore & length & eff_length & est_counts & TPM \\
\hline ADAR1 & TRINITY_DN2234624_c0_g1_i1 & $4 \mathrm{E}-26$ & 110 & 350 & 163.974 & 8 & 0.332477 \\
\hline ADAR2 & TRINITY_DN2234624_c0_g1_i1 & $9 \mathrm{E}-46$ & 163 & 350 & 163.974 & 8 & 0.332477 \\
\hline
\end{tabular}


neighbouring SINE content. The SINE insertions around these genes dated to the time of tandem $\mathrm{C}_{2} \mathrm{H}_{2}$ expansion, which contributed to the evolution of cephalopod neural complexity and morphological innovations. If this scenario is true, common traces of the RE burst should be found throughout the genome across all cephalopods. In the present analysis, we found distinct patterns of RE types in squid and octopus genome sequences. Ceph-SINEs, which are a type of tRNAderived SINEs originally isolated from squids (Akasaki et al. 2010), are found in common between squids and octopuses, but the frequency and patterns of those are different. Furthermore, Albertin et al. (2015) estimated that the burst of the REs was triggered between 25 and $56 \mathrm{Mya}$, which is far closer to the present compared with the timing of octopussquid separation ( $270 \mathrm{Mya})$, and hence not in accordance with the idea that all cephalopod experience common SINEbased genome rearrangements. The chromosome-based macrosynteny analysis using bivalve (scallop) genome revealed correspondence between the 19 scallop chromosomes, and 17 of them presumed ancestral bilaterian linkage groups (Wang et al. 2017). Therefore, numerous genome rearrangement found in octopus should have happened after the divergence between bivalves and octopus. Hence, it remains unclear when and how genomic rearrangement occurred in the cephalopod lineage. To narrow down the time range, chromosome-level comparative genomics based on longread sequencers should be carried out.

\section{Candidates for Bioluminescence in W. scintillans}

W. scintillans has ability to illuminate its body without the involvement of other organisms. Tsuji demonstrated that $W$. scintillans bioluminescence is produced by a luciferinluciferase reaction (Tsuji 1985, 2002). The responsible holoenzyme has not been characterized, but candidate proteins were found quite recently (Gimenez et al. 2016; Francis et al. 2017). In our independent study, we found two types of candidate genes in W. scintillans genome assembly sequence and RNA transcriptome, namely one similar to $P$. pyralis luciferase and the other similar to $S$. oualaniensis symplectin. As far as we know, there is no bioluminescent organism with two types of photoprotein.

Inamura et al. (1990) observed and Gimenez et al. (2016) pointed out that the luminescent colour of the arm emitter was slightly different from that of the body surface emitter. The arms emit bluish light, whereas the body surface emits slightly greenish light. Tsuji (2002) measured the spectral distribution of the light emitted from $W$. scintillans fourth arms and reported that it ranged from about 400 to $580 \mathrm{~nm}$ with a peak at $470 \mathrm{~nm}$ (blue). $\mathrm{He}$ also mentioned in the same paper that there had been a report by other workers that a small scattering of dermal organs on the ventral side had emitted a greenish-yellow light instead of blue light. It has been considered that the same molecular mechanism in different organs is used for the light emission in $W$. scintillans as suggested by Teranishi and Shimomura (2008).

$P$. pyralis luciferase emits greenish light. S. oualaniensis luciferase symplectin with coelenterazine was measured to emit light with wide spectral range (Chou et al. 2014), but Tsuji and Leisman (1981) measured the light spectrum of $S$. oualaniensis as a whole and found blue light emission around the wavelength of $455 \mathrm{~nm}$. These pieces of evidence made us speculate that the two different types of luciferase are employed to emit different wavelength light. One may suspect the existence of fluorescent protein in W. scintillans that can change the wavelength of the light derived from a single luciferase. We searched for the secondary bioluminescent proteins, such as green fluorescent proteins (GFPs), using animal GFPs as queries and found no apparent hit. Different types of substrate or slight difference in amino acid residues in the binding site of substrate is known to change the wavelength, and hence two different types of luciferase may not be needed for different colour in light.

As discussed by Francis et al. (2017), comparative genomics with $W$. scintillans can open a way to discuss evolution of luminescence genes. Luciferase enzymes have extremely varied structures, mechanisms and substrate specificities across extant organisms, and hence bioluminescence should have evolved independently at least 30 times across extant organisms (Hastings 1983). This study added new candidate sequences to the diversity of bioluminescence genes.

\section{Extensive RNA Editing in W. scintillans Transcripts}

Massive RNA editing was found in octopuses and squids tested so far, but not seen in the distant relative, such as the chambered nautilus and the sea hare (Liscovitch-Brauer et al. 2017). This is consistent with our analysis that shows W. scintillans transcriptome had extensive putative edit sites. The responsible genes, ADARs, were expressed in the same tissue, and it is reasonable to expect massive RNA editing with non-gene-specific manner. We expected that ecologically important enzymes such as the luciferases are necessary to finely tuned to the original functional complex and then avoided from the RNA editing. For example, proteins such as opsins are less variable to become adapted under certain environmental circumstances. Functionality of opsins appears to be maintained by purifying selection (Belcaid et al. 2019). Random variations by the frequent RNA editing may cause a malfunction despite purifying selection. However, RNA editing was also found in the W. scintillans wsluc1. The finding may support that the W. scintillans wsluc1 has still multifunction. The RNA editing is possibly fine-tuned in the cephalopods, characterizing that those genes may lead to find out DNA elements which positively/negatively control RNA editing machinery. 
Acknowledgements Samples were obtained with the courtesy of the Hotaruika Aquarium (Namerikawa, Toyama, Japan). We are also grateful for the support by Dr. Koji Okamura at the National Research Institute for Child Health and Development.

Funding The work by KY was supported by Grant-in-Aid for Scientific Research (B) [19H03200] from Japan Society for the Promotion of Science (JSPS). The faculty of Life and Environmental Sciences in Shimane University provided financial support for publishing this report.

Open Access This article is licensed under a Creative Commons Attribution 4.0 International License, which permits use, sharing, adaptation, distribution and reproduction in any medium or format, as long as you give appropriate credit to the original author(s) and the source, provide a link to the Creative Commons licence, and indicate if changes were made. The images or other third party material in this article are included in the article's Creative Commons licence, unless indicated otherwise in a credit line to the material. If material is not included in the article's Creative Commons licence and your intended use is not permitted by statutory regulation or exceeds the permitted use, you will need to obtain permission directly from the copyright holder. To view a copy of this licence, visit http://creativecommons.org/licenses/by/4.0/.

\section{References}

Akasaki T, Nikaido M, Nishihara H, Tsuchiya K, Segawa S, Okada N (2010) Characterization of a novel SINE superfamily from invertebrates: "Ceph-SINEs" from the genomes of squids and cuttlefish. Gene 454:8-19

Albertin CB, Simakov O, Mitros T, Wang ZY, Pungor JR, EdsingerGonzales E, Brenner S, Ragsdale CW, Rokhsar DS (2015) The octopus genome and the evolution of cephalopod neural and morphological novelties. Nature 524:220-224

Alon S, Garrett SC, Levanon EY, Olson S, Graveley BR, Rosenthal JJ, Eisenberg E (2015) The majority of transcripts in the squid nervous system are extensively recoded by A-to-I RNA editing. Elife 4

Bass BL, Weintraub H (1988) An unwinding activity that covalently modifies its double-stranded RNA substrate. Cell 55:1089-1098

Belcaid M, Casaburi G, McAnulty SJ, Schmidbaur H, Suria AM, Moriano-Gutierrez S, Pankey MS, Oakley TH, Kremer N, Koch EJ, Collins AJ, Nguyen H, Lek S, Goncharenko-Foster I, Minx P, Sodergren E, Weinstock G, Rokhsar DS, McFall-Ngai M, Simakov O, Foster JS, Nyholm SV (2019) Symbiotic organs shaped by distinct modes of genome evolution in cephalopods. Proc Natl Acad Sci U S A 116:3030-3035

Boersma YL, Newman J, Adams TE, Cowieson N, Krippner G, Bozaoglu K, Peat TS (2014) The structure of vanin 1: a key enzyme linking metabolic disease and inflammation. Acta Crystallogr Sect D Biol Crystallogr 70:3320-3329

Bray NL, Pimentel H, Melsted P, Pachter L (2016) Near-optimal probabilistic RNA-seq quantification. Nat Biotechnol 34:525-527

Capella-Gutiérrez S, Silla-Martínez JM, Gabaldón T (2009) trimAl: a tool for automated alignment trimming in large-scale phylogenetic analyses. Bioinformatics 25:1972-1973

Capozzi O, Carbone L, Stanyon RR, Marra A, Yang F, Whelan CW, de Jong PJ, Rocchi M, Archidiacono N (2012) A comprehensive molecular cytogenetic analysis of chromosome rearrangements in gibbons. Genome Res 22:2520-2528

Chou CM, Tung YW, Isobe M (2014) Molecular mechanism of Symplectoteuthis bioluminescence - Part 4: chromophore exchange and oxidation of the cysteine residue. Bioorganic Med Chem 22: $4177-4188$
Cole H, Reynolds TR, Lockyer JM, Buck GA, Denson T, Spence JE, Hymes J, Wolf B (1994) Human serum biotinidase. cDNA cloning, sequence, and characterization. J Biol Chem 269:6566-6570

da Fonseca RR, Couto A, Machado AM, Brejova B, Albertin CB, Silva F, Gardner P, Baril T, Hayward A, Campos A, Ribeiro ÂM, BarrioHernandez I, Hoving HJ, Tafur-Jimenez R, Chu C, Frazão B, Petersen B, Peñaloza F, Musacchia F, Alexander GC, Osório H, Winkelmann I, Simakov O, Rasmussen S, Rahman MZ, Pisani D, Vinther J, Jarvis E, Zhang G, Strugnell JM, Castro LFC, Fedrigo O, Patricio M, Li Q, Rocha S, Antunes A, Wu Y, Ma B, Sanges R, Vinar T, Blagoev B, Sicheritz-Ponten T, Nielsen R, Gilbert MTP (2020) A draft genome sequence of the elusive giant squid, Architeuthis dux. GigaScience 9

Darriba D, Posada D, Kozlov AM, Stamatakis A, Morel B, Flouri T (2020) ModelTest-NG: a new and scalable tool for the selection of DNA and protein evolutionary models. Mol Biol Evol 37:291-294

DePristo MA, Banks E, Poplin R, Garimella KV, Maguire JR, Hartl C, Philippakis AA, del Angel G, Rivas MA, Hanna M, McKenna A, Fennell TJ, Kernytsky AM, Sivachenko AY, Cibulskis K, Gabriel SB, Altshuler D, Daly MJ (2011) A framework for variation discovery and genotyping using next-generation DNA sequencing data. Nat Genet 43:491-498

dos Reis M, Thawornwattana Y, Angelis K, Telford MJ, Donoghue PC, Yang Z (2015) Uncertainty in the timing of origin of animals and the limits of precision in molecular timescales. Curr Biol 25:2939-2950

Emms DM, Kelly S (2015) OrthoFinder: solving fundamental biases in whole genome comparisons dramatically improves orthogroup inference accuracy. Genome Biol 16:157

Fallon TR, Lower SE, Chang CH, Bessho-Uehara M, Martin GJ, Bewick AJ, Behringer M, Debat HJ, Wong I, Day JC, Suvorov A, Silva CJ, Stanger-Hall KF, Hall DW, Schmitz RJ, Nelson DR, Lewis SM, Shigenobu S, Bybee SM, Larracuente AM, Oba Y, Weng JK (2018) Firefly genomes illuminate parallel origins of bioluminescence in beetles. Elife 7

Francis WR, Christianson LM, Haddock SHD (2017) Symplectin evolved from multiple duplications in bioluminescent squid. PeerJ 5:e3633

Fujii T, Ahn JY, Kuse M, Mori H, Matsuda T, Isobe M (2002) A novel photoprotein from oceanic squid (Symplectoteuthis oualaniensis) with sequence similarity to mammalian carbon-nitrogen hydrolase domains. Biochem Biophys Res Commun 293:874-879

Garrett S, Rosenthal JJC (2012) RNA Editing Underlies Temperature Adaptation in $\mathrm{K}+$ Channels from Polar Octopuses. Science 335: 848-851

Gimenez G, Metcalf P, Paterson NG, Sharpe ML (2016) Mass spectrometry analysis and transcriptome sequencing reveal glowing squid crystal proteins are in the same superfamily as firefly luciferase. Sci Rep 6

Gnerre S, Maccallum I, Przybylski D, Ribeiro FJ, Burton JN, Walker BJ, Sharpe T, Hall G, Shea TP, Sykes S, Berlin AM, Aird D, Costello M, Daza R, Williams L, Nicol R, Gnirke A, Nusbaum C, Lander ES, Jaffe DB (2011) High-quality draft assemblies of mammalian genomes from massively parallel sequence data. Proc Natl Acad Sci U S A 108:15131518

Goto T, Iio H, Inoue S, Kakoi H (1974) Squid bioluminescence I. Structure of Watasenia oxyluciferin, a possible light-emitter in the bioluminescence of Watasenia scintillans. Tetrahedron Lett. https:// doi.org/10.1016/S0040-4039(01)92245-2

Gregory TR (2019) Animal Genome Size Database. http://www. genomesize.com. Accessed 6 May 2019

Hara T, Hara R (1980) Retinochrome and rhodopsin in the extraocular photoreceptor of the squid, Todarodes. J Gen Physiol 75:1-19

Hastings JW (1983) Biological diversity, chemical mechanisms, and the evolutionary origins of bioluminescent systems. J Mol Evol 19:309-321 
Hayashi K, Kawai YL, Yura K, Yoshida MA, Ogura A, Hata K, Nakabayashi K, Okamura K (2016) Complete genome sequence of the mitochondrial DNA of the sparkling enope squid, Watasenia scintillans. Mitochondrial DNA A DNA Mapp Seq Anal 27:1842-1843

Hijikata A, Yura K, Noguti T, Go M (2011) Revisiting gap locations in amino acid sequence alignments and a proposal for a method to improve them by introducing solvent accessibility. Proteins Struct Funct Bioinf 79:1868-1877

Inamura O, Kondoh T, Ohmori K (1990) Observations on minute photophores of the firefly squid, Watasenia scintillans. Science report of the Yokosuka City Museum 38:101-105

Inoue S, Sugiura S, Kakoi H, Inoue S, Sugiura S, Kakoi H, Hasizume K, Goto T, Iio H (1975) Squid bioluminescence II. Isolation from Watasenia scintillans and synthesis of 2-(p-Hydroxybenzyl)-6-( $p$ hydroxyphenyl)-3,7-dihydroimidazo[1,2-a]pyrazin-3-one. Chem Lett. https://doi.org/10.1246/cl.1975.141

Inoue S, Kakoi H, Goto T (1976) Squid bioluminescence III. Isolation and structure of Watasenia luciferin. Tetrahedron Lett 17:29712974

Isobe M, Kuse M, Tani N, Fujii T, Matsuda T (2008) Cysteine-390 is the binding site of luminous substance with symplectin, a photoprotein from Okinawan squid, Symplectoteuthis oualaniensis. Proc Japan Acad Ser B 84:386-392

Kinjo AR, Bekker G-J, Wako H, Endo S, Tsuchiya Y, Sato H, Nishi H, Kinoshita K, Suzuki H, Kawabata T, Yokochi M, Iwata T, Kobayashi N, Fujiwara T, Kurisu G, Nakamura H (2018) New tools and functions in data-out activities at Protein Data Bank Japan (PDBj). Protein Sci 27:95-102

Kohany O, Gentles AJ, Hankus L, Jurka J (2006) Annotation, submission and screening of repetitive elements in Repbase: RepbaseSubmitter and Censor. BMC Bioinforma 7:474

Kozlov AM, Darriba D, Flouri T, Morel B, Stamatakis A (2019) RAxML-NG: A fast, scalable, and user-friendly tool for maximum likelihood phylogenetic inference. Bioinformatics 35:4453-4455

Kröger B, Vinther J, Fuchs D (2011) Cephalopod origin and evolution: a congruent picture emerging from fossils, development and molecules: extant cephalopods are younger than previously realised and were under major selection to become agile, shell-less predators. BioEssays 33:602-613

Lander ES, Linton LM, Birren B et al (2001) Initial sequencing and analysis of the human genome. Nature 409:860-921

Li H, Handsaker B, Wysoker A, Fennell T, Ruan J, Homer N, Marth G, Abecasis G, Durbin R, 1000 Genome Project Data Processing Subgroup (2009) The sequence alignment/map (SAM) format and SAMtools. Bioinformatics 25:2078-2079

Liscovitch-Brauer N, Alon S, Porath HT, Elstein B, Unger R, Ziv T, Admon A, Levanon EY, Rosenthal JJC, Eisenberg E (2017) Trade-off between transcriptome plasticity and genome evolution in cephalopods. Cell 169:191-202.e11

Marçais G, Kingsford C (2011) A fast, lock-free approach for efficient parallel counting of occurrences of k-mers. Bioinformatics 27:764770

Murakami M, Kouyama T (2008) Crystal structure of squid rhodopsin. Nature 453:363-367

Oliver KR, Greene WK (2009) Transposable elements: powerful facilitators of evolution. BioEssays 31:703-714

Picardi E, Pesole G (2013) REDItools: high-throughput RNA editing detection made easy. Bioinformatics 29:1813-1814

Pinto Y, Cohen HY, Levanon EY (2014) Mammalian conserved ADAR targets comprise only a small fragment of the human editosome. Genome Biology 15:R5

Quinlan AR, Hall IM (2010) BEDTools: a flexible suite of utilities for comparing genomic features. Bioinformatics 26:841-842
Rambaut A (2009) FigTree version 1.4.2 [computer program]. https:// github.com/rambaut/figtree

Šali A, Blundell TL (1993) Comparative protein modelling by satisfaction of spatial restraints. J Mol Biol 234:779-815

Schwiening CJ (2012) A brief historical perspective: Hodgkin and Huxley. J Physiol 590:2571-2575

Seidou M, Sugahara M, Uchiyama H, Hiraki K, Hamanaka T, Michinomae M, Yoshihara K, Kito Y (1990) On the three visual pigments in the retina of the firefly squid, Watasenia scintillans. J Comp Physiol A 166

Shen MR, Batzer MA, Deininger PL (1991) Evolution of the master Alu gene(s). J Mol Evol 33:311-320

Shichida Y, Matsuyama T (2009) Evolution of opsins and phototransduction. Philos Trans R Soc B Biol Sci 364:2881-2895

Simakov O, Marletaz F, Cho SJ, Edsinger-Gonzales E, Havlak P, Hellsten U, Kuo DH, Larsson T, Lv J, Arendt D, Savage R, Osoegawa K, de Jong P, Grimwood J, Chapman JA, Shapiro H, Aerts A, Otillar RP, Terry AY, Boore JL, Grigoriev IV, Lindberg DR, Seaver EC, Weisblat DA, Putnam NH, Rokhsar DS (2013) Insights into bilaterian evolution from three spiralian genomes. Nature 493:526-531

Simão FA, Waterhouse RM, Ioannidis P, Kriventseva EV, Zdobnov EM (2015) BUSCO: assessing genome assembly and annotation completeness with single-copy orthologs. Bioinformatics 31:3210-3212

Stanke M, Diekhans M, Baertsch R, Haussler D (2008) Using native and syntenically mapped cDNA alignments to improve de novo gene finding. Bioinformatics 24:637-644

Teranishi K, Shimomura O (2008) Bioluminescence of the arm light organs of the luminous squid Watasenia scintillans. Biochim Biophys Acta Gen Subj 1780:784-792

Trapnell C, Pachter L, Salzberg SL (2009) TopHat: discovering splice junctions with RNA-Seq. Bioinformatics 25:1105-1111

Tsuji FI (1985) ATP-dependent bioluminescence in the firefly squid, Watasenia scintillans. Proc Natl Acad Sci USA 82:4629-4632

Tsuji FI (2002) Bioluminescence reaction catalyzed by membrane-bound luciferase in the "firefly squid," Watasenia scintillans. Biochim Biophys Acta Biomembr 1564:189-197

Tsuji FI (2005) Role of molecular oxygen in the bioluminescence of the firefly squid, Watasenia scintillans. Biochem Biophys Res Commun 338:250-253

Tsuji FI, Leisman GB (1981) K+/Na+-triggered bioluminescence in the oceanic squid Symplectoteuthis oualaniensis. Proc Natl Acad Sci U S A 78: $6719-6723$

Vinther J, Sperling EA, Briggs DEG, Peterson KJ (2012) A molecular palaeobiological hypothesis for the origin of aplacophoran molluscs and their derivation from chiton-like ancestors. Proc R Soc B Biol Sci 279:1259-1268

Vurture GW, Sedlazeck FJ, Nattestad M, Underwood CJ, Fang H, Gurtowski J, Schatz MC (2017) GenomeScope: fast reference-free genome profiling from short reads. Bioinformatics 33:2202-2204

Wang S, Zhang J, Jiao W, Li J, Xun X, Sun Y, Guo X, Huan P, Dong B, Zhang L, Hu X, Sun X, Wang J, Zhao C, Wang Y, Wang D, Huang X, Wang R, Lv J, Li Y, Zhang Z, Liu B, Lu W, Hui Y, Liang J, Zhou Z, Hou R, Li X, Liu Y, Li H, Ning X, Lin Y, Zhao L, Xing Q, Dou J, Li Y, Mao J, Guo H, Dou H, Li T, Mu C, Jiang W, Fu Q, Fu X, Miao Y, Liu J, Yu Q, Li R, Liao H, Li X, Kong Y, Jiang Z, Chourrout D, Li R, Bao Z (2017) Scallop genome provides insights into evolution of bilaterian karyotype and development. Nat Ecol Evol 1

Wiederstein M, Sippl MJ (2007) ProSA-web: interactive web service for the recognition of errors in three-dimensional structures of proteins. Nucleic Acids Res 35:W407-W410

Yoshida MA, Ishikura Y, Moritaki T, Shoguchi E, Shimizu KK, Sese J, Ogura A (2011) Genome structure analysis of molluscs revealed 
whole genome duplication and lineage specific repeat variation. Gene 483:63-71

Young JZ (1971) The anatomy of the nervous system of octopus vulgaris. Clarendon Press, Oxford, p 690

Young RE, Roper CFE (1977) Intensity regulation of bioluminescence during countershading in living midwater animals. Science 191: 1046-1048

Young RE, Roper CFE, Walters JF (1979) Eyes and extraocular photoreceptors in midwater cephalopods and fishes: their roles in detecting downwelling light for counterillumination. Mar Biol 51: 371-380

Yu Y, Zhou H, Kong Y, Pan B, Chen L, Wang H, Hao P, Li X (2016) The landscape of A-to-I RNA editome is shaped by both positive and purifying selection. PLoS Genet 12:e1006191

Publisher's Note Springer Nature remains neutral with regard to jurisdictional claims in published maps and institutional affiliations. 